\title{
Electro-Active Polymer (EAP) "Dimple" Actuators For Flow Control: Design and Characterisation.
}

\author{
Stella S. Dearing*, Jonathan F. Morrison, Lorenzo Iannucci \\ Department of Aeronautics, Imperial College, SW7 2AZ, London, England
}

\begin{abstract}
The long-term goal of this work is the development of a 'smart' skin capable of sensing and real-time actuation of oncoming turbulent flow, specifically to reduce skin-friction drag. Active "dimples" are time-dependent depressions that optimally alter local flow conditions [2]. Lightweight Electro-Active Polymers (EAPs) are ideal for these control surfaces since they offer high strain rate, fast response, low power consumption and ease of manufacture [13]. Key features for integration into a control system are robustness and repeatable, predictable motion. For this objective dimples are assessed and values for out-of-plane deflection, frequency response and vibrational modes, failure modes as well as power consumption values are presented. The dimple design comprises a buckling actuator, which can actuate without the need of a directional bias, differentiating itself from previous designs [16, 17, 18, $19,20,21]$. It also actuates from flush to the surface providing asymmetrical forcing, a key consideration for flow control purposes. It is shown that the dimple actuates with vibrational modes higher than the fundamental, and that these are non-linear, an important consideration for this type of device. The power consumption of the device, as required for net power savings, and device robustness is promising.
\end{abstract}

Key words: Electro-active polymers (EAPs), buckling diaphragm

\footnotetext{
*Corresponding author. Present Address: Dipartimento di Energetica e Macchine, Università di Udine, Via delle Scienze 204, Udine 33100, Italy. Tel: +39 0432558006 ; Fax: +390432 558025

Email addresses: stella.dearing@uniud.it (Stella S. Dearing ), j.morrison@ic.ac.uk (Jonathan F. Morrison ), lo.ianucci@ic.ac.uk (Lorenzo Iannucci)
} 
actuators, power consumption, mechanical, electromechanical characterisation

\section{Introduction}

Commercial aircraft, at typical cruise conditions, fly in the turbulent regime where skin-friction accounts for $50 \%$ of the total drag [5]. There is, therefore, significant environmental and economical incentive to reduce skin friction drag. Active "dimples" act as time dependent depressions that inject local flow disturbances capable of interacting with structures existing in the turbulent boundary layer. These structures are 10-1000 $\mu \mathrm{m}$ in size, and occur at frequencies of $0.1-1 \mathrm{kHz}$ and in the recent studies [6] "dimples" are actuated with out-of-plane deflections 5\%-10\% of the dimple diameter. However, their application necessitates a good understanding of dimple-fluid interaction hence, as a first step, "mesoscale" devices are studied in laminar boundary layers. This requires working knowledge of the dimple motion and surface characteristics during actuation and an actuator that can fulfil the necessary criteria. Even though proof-of-concept devices do not need the stringent requirements outlined above, the design must be readily miniaturised and capable of achieving these specifications.

The dimple design uses dielectric EAPs as the actuating mechanism. They provide unique benefits in terms of manufacturing and performance compared to more established technologies such as piezo-ceramics and mechanically driven actuators. They are lightweight, flexible, offer fast response, high strain rate and provide low power consumption required for net energy savings $[13,10,11,12]$. Furthermore, they exhibit the reciprocal mechanism for sensing, hence are well suited for fabrication as a 'smart' skin, a checkerboard of intelligent and interactive wall sensors and actuators on a continuous surface, which is envisaged for the control of turbulence $[1,9]$.

The working principle of a dielectric EAP is based on the compression of an elastomer film, by electrostatic pressure, also referred to as Maxwell Stress, that is applied by compliant electrodes upon the application of an electric field. By virtue of incompressibility, the compression of the elastomer results in an elongation of the membrane which is used for actuation, as shown in Fig. 1. Actuators of this type are referred to as dielectric elastomer actuators $(\mathrm{DE} \overline{\mathrm{As}})$. 

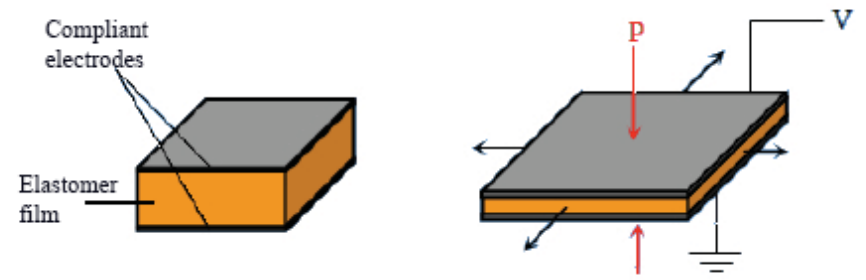

Figure 1: Principle of Actuation [2].

One of the main challenges of EAP technology is that it is largely unestablished, since they consist of non-linear materials that need to be suitably characterised [8] and modelled to be used with predictable and repeatable motion as required for a flow control device [5]. Moreover the application requires a good understanding of dimple motion (deflection, velocity and acceleration of the dimple surface) and surface topology characteristics. The scope of this work is to present the design a proof-of-concept mesoscale device and assess it in terms of frequency response, vibrational modes and outof-plane behaviour, actuator robustness, actuation repeatability and power consumption of the device.

\section{Actuator Design}

\subsection{Operating Principle}

An EAP is essentially a compliant capacitor that consists of an elastomer dielectric, with thickness typically $100 \mu \mathrm{m}$ (to minimise applied voltages), sandwiched between two compliant electrodes, as shown in Fig. 1. On ap-

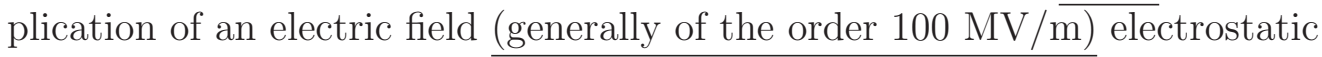
attraction between the two oppositely charged electrodes causes a mechanical compression in the thickness direction whilst the repulsion of like charges on the same electrode causes the surface to the expand. The driving force for actuation, the electrostatic pressure, can be written as

$$
P=\epsilon_{o} \epsilon_{r} \frac{V^{2}}{z_{o}^{2}},
$$

where $\epsilon_{r}$ is the dielectric constant, $\epsilon_{o}$ is the permittivity of a vacuum, $V$ is the voltage across the electrodes, and $z_{o}$ is the undeformed elastomer thickness, with the assumption that there is a constant electric field across the electrodes. Thickness strain, based on Hooke's law can be written as

$$
S_{z}=\frac{\epsilon_{o} \epsilon_{r}}{E} \frac{V^{2}}{z_{o}^{2}}
$$


assuming free boundary conditions. $E$ is the Young's modulus of the material, a constant which describes the stiffness of the material. It has been suggested $[13,15]$ that, for thickness strains of less than 10\%, Equation 2 holds true. This assumption holds for the current dimple design, since the maximum calculated thickness strain was less than $5 \%$ for the deflections observed. Thickness strain is related to in-plane strain through the incompressibility relation

$$
\left(1+S_{x}\right)\left(1+S_{y}\right)\left(1+S_{z}\right)=1,
$$

where $S$ refers to strain and subscripts $x, y$ and $z$ are Cartesian coordinates. For the unconstrained case $S_{x}=S_{y}=S_{r}$ and Equation 3 can be written as

$$
\left(1+S_{z}\right)\left(1+S_{r}\right)^{2}=1 .
$$

Equation 2 highlights the relationship between Young's modulus and thickness strain. This equation, with the incompressibility constraint, Equation 4, provides a means of calculating the radial strain. For a biaxially constrained design, as is the dimple case, as long as the region away from the edge is considered, the assumption that $S_{x}=S_{y}$ holds true. This assumption has been confirmed for similar-sized configurations. Bubble inflation tests of the material under study were carried out and radial strain was measured using Digital Speckle Photogrammetry (DSP). Bubble inflation tests involve the application of a differential pressure across a membrane which is clamped at its circumference. Owing to the similarity in the boundary conditions, this biaxial loading can be considered to be the same as that found during the dimple actuation. During inflation, the measured radial strain was found to be the same in both directions.

\subsection{Dimple Design}

Depending on the boundary conditions of the elastomer film, upon actuation, the membrane either buckles, bends or elongates. In the case of the diaphragm actuator, membranes are clamped at a desired radius that defines the size of the actuator. When an electric field is applied, the compression of the film causes in-plane expansion. Since the membrane periphery is fixed, no in-plane movement can occur and isotropic compressive stress builds up to a critical buckling limit, dependent on the membrane mechanical properties and geometry. Out-of-plane deflection then takes place with the formation of a bump or depression depending on the directional bias in the form of an 
external force $[27,17]$ or asymmetry in the structure [21]. The elastomer film is clamped only on one side, such that, with a proper mount, the dimple actuator lies flush to the surface and no aerodynamic effect occurs. Fig. 2(a) shows out-of-plane buckling of a diaphragm actuator.

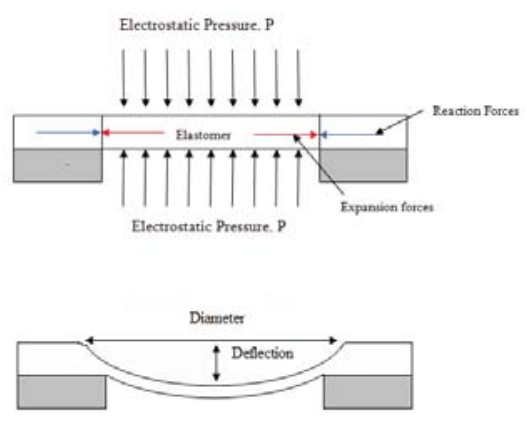

(a)

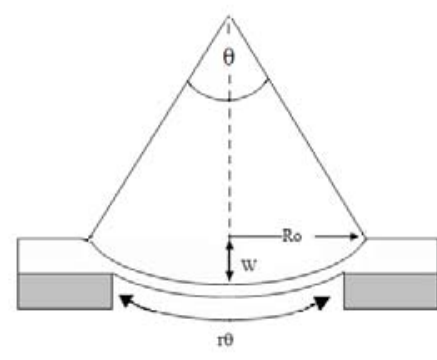

(b)

Figure 2: (a) Principle of actuation [2], \& (b) Geometrical relationships of a deflected dimple.

\subsection{Directional Bias}

The dimple was forced with one electrode is held at ground and the other electrode is forced by an alternating current in the positive quadrant (hence going from $+0 \mathrm{kV}$ to the required driving voltage and then switching). In this configuration it is found that there is an asymmetry in the forcing, causing the biasing effect. The preferential direction of deflection was found to be directly related to the location of the ground electrode. This may be a result of an imbalance of charges caused by this configuration. Since the total forces comprise electrostatic attraction between oppositely charged electrodes and repulsion between the same charges on the same electrode, if an imbalance of charge between the two electrodes is generated then the repulsion forces at each electrode would no longer be equal. Using this technique the dimple can be switched to a bump by simply reversing the position of the grounded electrode across the elastomer film.

\subsection{Out-of-plane deflections}

The out-of plane deflection can be estimated using analysis based on geometric principles. This analysis assumes that the shape of the deflection 
is spherical hence the radius of curvature of a sphere can be written as

$$
d s=\int_{-R_{0}}^{R_{0}}\left[\left(\frac{d w}{d r}\right)^{2}+1\right]^{\frac{1}{2}} d r^{1}
$$

with

$$
s=2\left(1+S_{r}\right) R_{0}=r \theta,
$$

where $s$ is the arc length of circle of radius $r$ extended through an angle $\theta$ and $R_{0}$ the original dimple radius, shown schematically Fig. 2(b). Equation 5 uses the estimated radial strain for a linear material with free boundary conditions, $S_{r}$, which can be estimated using Equation 4 or measured to calculate the radius of curvature. From trigonometry, the original dimple radius, $R_{0}$ is written as

$$
R_{0}=r \sin \left(\frac{\theta}{2}\right)
$$

Substituting for $R_{0}$ in Equation 7 and using a Taylor series expansion:

$$
\theta=\sqrt{24\left(1-\frac{1}{\left(1+S_{r}\right)}\right)} .
$$

This is used to calculate the out-of-plane deflection given by

$$
w=\frac{R_{0}}{\sin \left(\frac{\theta}{2}\right)}\left[1-\cos \left(\frac{\theta}{2}\right)\right] .
$$

\section{Actuator Fabrication}

\subsection{Material Selection}

Any elastomer can be used as the dielectric if it is non conducting, making it possible to tailor the material properties to suit the needs of the application [23]. These devices must be designed to be able to withstand in-flight operating temperatures $\left(-80^{\circ} \mathrm{C}\right.$ to $\left.100^{\circ} \mathrm{C}\right)$ and various weather conditions. Specifically it is required that they should be able to resist moisture and not degrade with UV heating nor with repeated heating or cooling. Generally silicone and acrylic are the most commonly used DEA materials

${ }^{1}$ See [33] for derivation 
as result of superior performance compared to the alternatives. However, acrylic does not possess the thermal stability and good frequency response [28] required for the present applications. Silicone retains room temp characteristics across a wide range of temperatures $\left(-100^{\circ} \mathrm{C}\right.$ to $\left.+316^{0}\right)$, and is thus suitable for in-flight conditions. It also exhibits a lower viscoelastic response than acrylic and a minimal time-history dependence [28]. Furthermore it is resistant to the external environment including moisture and is known to be very stable towards UV-radiation from sunlight [29].

Equation 2 shows that DEA performance is dependent on the material dependent properties, E and $\epsilon_{r}$. Equation 2, however, is unable to capture another limiting factor in performance; the material's dielectric breakdown strength. In fact, performance is a compromise between the material dielectric strength and the ratio of $\frac{\epsilon_{r}}{E}$ [30]. Materials of higher Shore A number (a measure of hardness), are generally stiffer. However, they also have higher breakdown strengths. Three materials of Shore A numbers of 5, 30 and 50 have been compared( see [30] for more details). The material with a Shore A number of 30 provides the best performance in terms of strain and breakdown strength. For this reason all dimples are designed using Nusil MED4930 as the dielectric.

The dielectric breakdown strength for $M E D 4930$ was found to be $46 \mathrm{MV} / \mathrm{m}$, using a simple electro-mechanical test. A piece of unstretched elastomer is placed uniformly over a frame and a circular electrode deposited on the film. An electric field was then applied and the film between the electrodes contracted in thickness and expanded in area. When dielectric breakdown occurred the material burned. By increasing the voltage until breakdown, the dielectric breakdown strength was measured. The dielectric constant was measured as $2.8 \mathrm{MV} / \mathrm{m}$ (within $10 \%$ accuracy) using a simple experimental rig consisting of two rigid electrodes which were connected to a sensitive capacitance meter. The dielectric was sandwiched between the two rigid electrodes and the capacitance was measured. The dielectric constant was worked out using the relation for capacitance of a parallel plate capacitor: $C=\epsilon_{o} \epsilon_{r} \frac{A}{d}$, where $C$ is the capacitance, $A$ is the area of overlap of the two plates measured in $m^{2}, \epsilon_{r}$ is the dielectric constant, $\epsilon_{o}$ the permittivity of free space and $d$ is the separation between the plates. MED4930 is a PDMS-VMQ silicone, with a specific gravity of 1.13 , Shore A of 30 and produces a strain $200 \%$ at 1.2 MPa stress (a common performance measure for elastomers).

Graphite dust electrodes are used to conduct charge onto the dielectric surface. These electrodes can be easily applied; they are either stencilled 


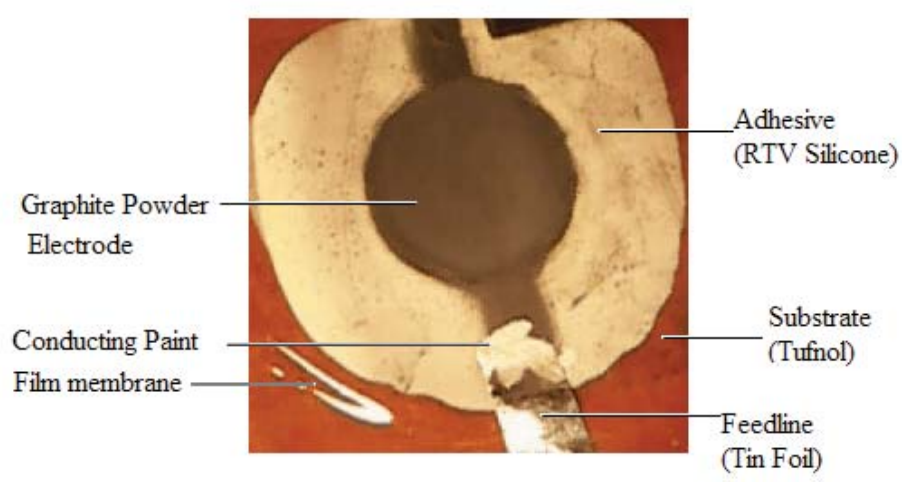

Figure 3: Dimple Assembly.

or brushed on. They have low density and have the ability to follow the dielectric surface without restriction. The MED4930 surface is already tacky and the electrodes adhere well to the surface. Although the resistance of the electrodes increases at high strains, these are not necessary for dimple actuation so that it was not necessary to take this into account during these experiments.

\subsection{Dimple Fabrication}

Circular dimples are fabricated using $100 \mu \mathrm{m}$ film of MED4930 and graphite electrodes, with radii of $5 \mathrm{~mm}, 10 \mathrm{~mm}$ and $15 \mathrm{~mm}$. The design comprises a substrate with an orifice of the same diameter as the actuator and a thin elastomeric film. The film is stuck onto the substrate, thus enforcing the boundary condition at the edge of the orifice. The elastomer films are fabricated commercially, the finished product available in rolls of elastomer film cured onto a backing material. The films are stuck onto the substrate with a suitable glue (which should be silicone-based); in this case silicone RTV (Dow Corning 'Toray': 1-part alcohol system SE 9187) was used. Once the glue is cured the backing material is peeled. Prior to deposition of the membrane, feed lines, made from tinfoil, are laid down to connect the bottom electrode to the power cable. The top and the bottom electrode are stencilled on using graphite powder and a cotton bud. The upper feed line is placed on the upper surface and silver conducting paint is used to ensure contact between the feed line and the graphite powder. Fig. 3 shows a fabricated assembly. 

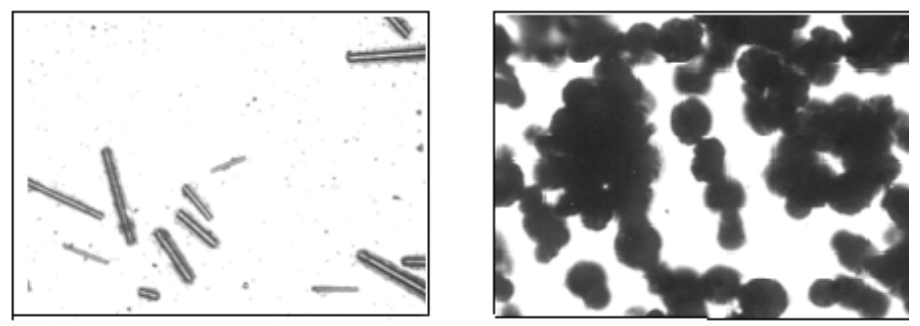

Figure 4: Left hand side: Nusil R1505 carbon-loaded conducting elastomer. The carbon fibre fragments are clearly visible. Note the wide spacing between fragments which prevents electrical conduction. Right hand side: Nusil CV2646 silver-loaded conducting elastomer. The silver particles are seen. Typical particle diameter is 6 microns

An electric field is generated across the membrane using graphite electrodes in the form of graphite powder (West System Products 423). The graphite consists of particles sizes in the range of a few to $50 \mu \mathrm{m}$ in diameter. The elastomer films are already tacky, hence the adherence between the graphite particles and the film is very good. It was found that neither the appearance of the graphite electrodes nor the performance of the dimple deteriorated with time.

As alternatives to graphite electrodes, both rubber electrodes and grease electrodes were investigated. However the graphite electrodes were preferred. $40 \mu \mathrm{m}$ carbon-loaded and silver-loaded elastomer electrodes were made using spin coating, to ensure thin layer and uniform deposition. It was found that the conducting particles were spread too thinly and hence unsuitable as electrodes- This can be seen from scanning electron images of the spin-coated electrodes, Fig. 4. Carbon grease electrodes were also tested. These were made from conductive graphite suspended in silicone oil. It was found that grease electrodes were messy to work with, and can be spread by contact with other surfaces. They also tend to collect dust and other particulates with time. Furthermore it was found the unstretched silicone diaphragms swell with time under the weight of the electrode grease. Graphite electrodes, on the other hand, provide a fast and cheap way of fabricating electrodes and also ensure constant uniformity with a negligible increase in the stiffness of the membrane. Graphite electrodes are also fully compliant as the particles are free to move with the elastomer. 


\section{Actuator Performance}

\subsection{Experimental Setup}

Dimples are excited by keeping one electrode at ground while the voltage on the other is varied from zero to a maximum permissible (positive) voltage: a.c. excitation was provided using both sine and square waves over a range of frequencies. The dimple is excited using a high voltage amplifier, model 609-E and current measurements are taken using a shunt resistor ${ }^{2}$ permitting the indirect measurement of the current using a high-voltage probe (LeCroy PPE 6kV).

Out-of-plane deflection measurements are taken using a topological scanner which consists of a laser-meter ${ }^{3}$ attached to a $x, z$ traverse driven by a computer through a data acquisition card, DT9803. This permits the automated capture of the $x, y, z$ coordinates of the dimple surface. Phase averages of maximum deflection are calculated for the $\epsilon / D$ results presented below.

The frequency response of the dimples is measured by using the topological scanning unit and fixing it at the dimple midpoint and forcing the actuator between 1-100 $\mathrm{Hz}$ at electric field strengths between $28 \mathrm{MV} / \mathrm{m}(2.8$ $\mathrm{kV})$ and $42 \mathrm{MV} / \mathrm{m}(4.2 \mathrm{kV})$. The resonant modes and mode shapes are investigated using a Laser Doppler Vibrometer (LDV), Polytec PSV-400, because it can carry out measurements and process data faster than the topological scanning unit and provide better resolution, in the $n m$ range. A $0-2.0 \mathrm{kV}$ and $0-3.0 \mathrm{kV}$ sinusoidal voltage was applied between the electrodes while the frequency of the a.c. signal was swept between $5 \mathrm{~Hz}$ and $5 \mathrm{kHz}$.

\subsection{Out-of-Plane Deflections}

Fig. 5 shows the maximum depth-to-diameter ratios, experimentally determined using a laser displacement meter, Microepsilon opto NCDT-2200, compared to theoretical values determined from Equation 9. It was found that dimple performance is sensitive to the dimple diameter; Fig. 5 shows that dimples of $5 \mathrm{~mm}$ radius achieve the maximum depth-to-diameter ratio. On

\footnotetext{
${ }^{2}$ a conducting element bridge across a circuit or a portion of a circuit, establishing a current path auxiliary to the main circuit

${ }^{3}$ The laser meter, Microepsilon opto NCDT-2200, has a measuring range of $2 \mathrm{~mm}$ over $10 \mathrm{~V}$, that is an accuracy of $0.2 \mu \mathrm{m}$ per millivolt, a resolution of $0.1 \mu \mathrm{m}$ and a measuring rate of $10 \mathrm{kHz}$. The traverse is calibrated to $2.5 \mu \mathrm{m}$ per step.
} 
the other hand, larger diameters tend to be less robust than the smaller one; the smallest dimple can withstand a higher voltage than the larger ones and can reliably perform for longer without failing. In fact the $5 \mathrm{~mm}$ dimple was used up to $4.8 \mathrm{kV}$ and could be cycled reliably for 10800 cycles (duration of one scan). At best, it could withstand 60 times that (the dimple actuator was used continuously for one and a half months without failing or degradation to performance).

The discrepancy between the theory outlined in $\S 2.4$ and experimental data, especially in the larger dimples, may be explained by the presence of wrinkling. According to Stein-Hedgepeth theory [31] all compressive stresses are eliminated completely by formation of wrinkling. Since the theory outlined in $§ 2.4$ describes the behaviour of the dimple under stress, if wrinkling occurs it is no longer valid. Generally, membranes will take one of following three states: taut, slack or regularly wrinkled. In this case of a circular plate, the equation for critical buckling compressive stress, $\sigma_{c r}$, is used[34]:

$$
\sigma_{c r}=\kappa \frac{E}{1-\nu^{2}}\left(\frac{t}{r}\right)^{2}
$$

where $E$ is Young's modulus estimated from Equation 1 using a linear Hookean relation, $t$ is the thickness, $r$ is dimple radius, $\nu$ is Poisson's ratio and $\kappa=0.35$ for simply supported boundary conditions and $\kappa=1.22$ for built-in boundary conditions.

The plots in Fig. 6 illustrate the initial imperfection, buckling, defined as the point at which the initial portion of the plot becomes non-linear. This definition is only relevant to linear elastic material behaviour, an underlining assumption in the derivation of the simple dimple theory. The critical buckling stress for each dimple size is calculated using Equation 10. Then the equivalent buckling voltage is found by equating the critical buckling stress (Equation 10) to the electrostatic pressure (Equation 1): $V_{c r}=\sqrt{\frac{\sigma_{c r} t^{2}}{\epsilon_{r} \epsilon_{o}}}$. It is found that the critical buckling voltage is predicted to decrease with increasing radius size: that is for the $5 \mathrm{~mm}, 10 \mathrm{~mm}$ and $15 \mathrm{~mm}$ dimples, they are respectively, $1366.58 \mathrm{~V}, 683.00 \mathrm{~V}$ and $455.86 \mathrm{~V}(13.66 \mathrm{MV} / \mathrm{m}, 6.83 \mathrm{MV} / \mathrm{m}, 4.55 \mathrm{MV} / \mathrm{m}$,). However, Fig. 6, a-c, show the opposite trend: the critical buckling voltage increases with radius size: $2.0 \mathrm{kV}, 3.0 \mathrm{kV}$ and $3.5 \mathrm{kV}(20 \mathrm{MV} / \mathrm{m}, 25 \mathrm{MV} / \mathrm{m}$, $30 \mathrm{MV} / \mathrm{m}$ ) respectively. Once initial buckling occurs (based on Equation 10), in the post-buckled state it will still be possible to be in a state of compression which could exceed the wrinkling stress level within the material. This 


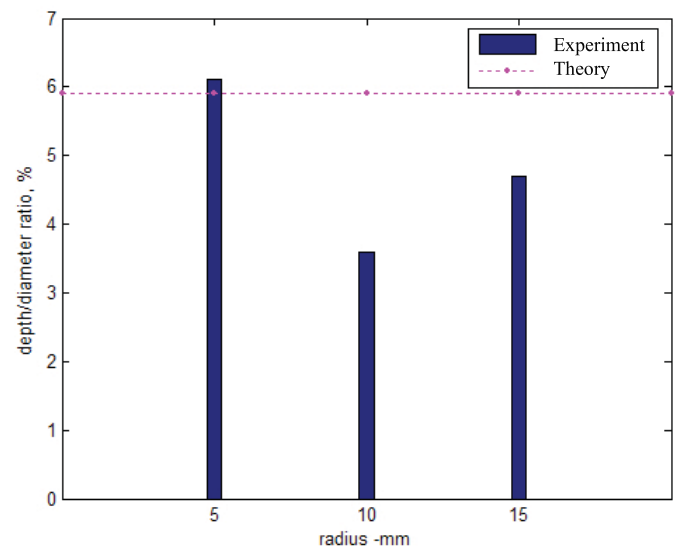

Figure 5: Summary of maximum depth to diameter ratios for different sized dimples at $4.4 \mathrm{MV} / \mathrm{m}$.

will partially or fully eliminate the compressive stresses. Therefore before out-of-plane buckling can occur a greater radial strain must be achieved: thus the critical buckling voltage will appear to be higher. However, further studies are required to determine the appropriate wrinkling stress level and their wavelengths. For example, a detailed non-linear Finite Element analysis could be used to model the initial buckling, post-buckling behaviour and ultimate wrinkling of the membrane, if a wrinkling stress level is reached.

Another interesting feature from Fig. 6, a-c, is the downward and upward part of the displacement versus voltage cycle. Except for the 5-mm dimple, the initial part of the upward part of the cycle is the same as that for the initial downward part of the cycle. After buckling (and beyond) the actuator response shows hysteresis, behaving differently for downward and upward cycles: for the same displacement, a lower voltage is required on the upward part of the cycle than on the downward part. From examination of a single trace, as shown in Fig. 7 , it is clear that this hysteresis is not due to an asymmetry between the displacement curve for the upward and downward motion of the dimple (about the maximum or minimum displacement the displacement curves either side are the same). However, there is a phase shift between the dimple displacement and driving voltage which is most significant for the 5-mm dimple, as shown from $\S 4.3$. 

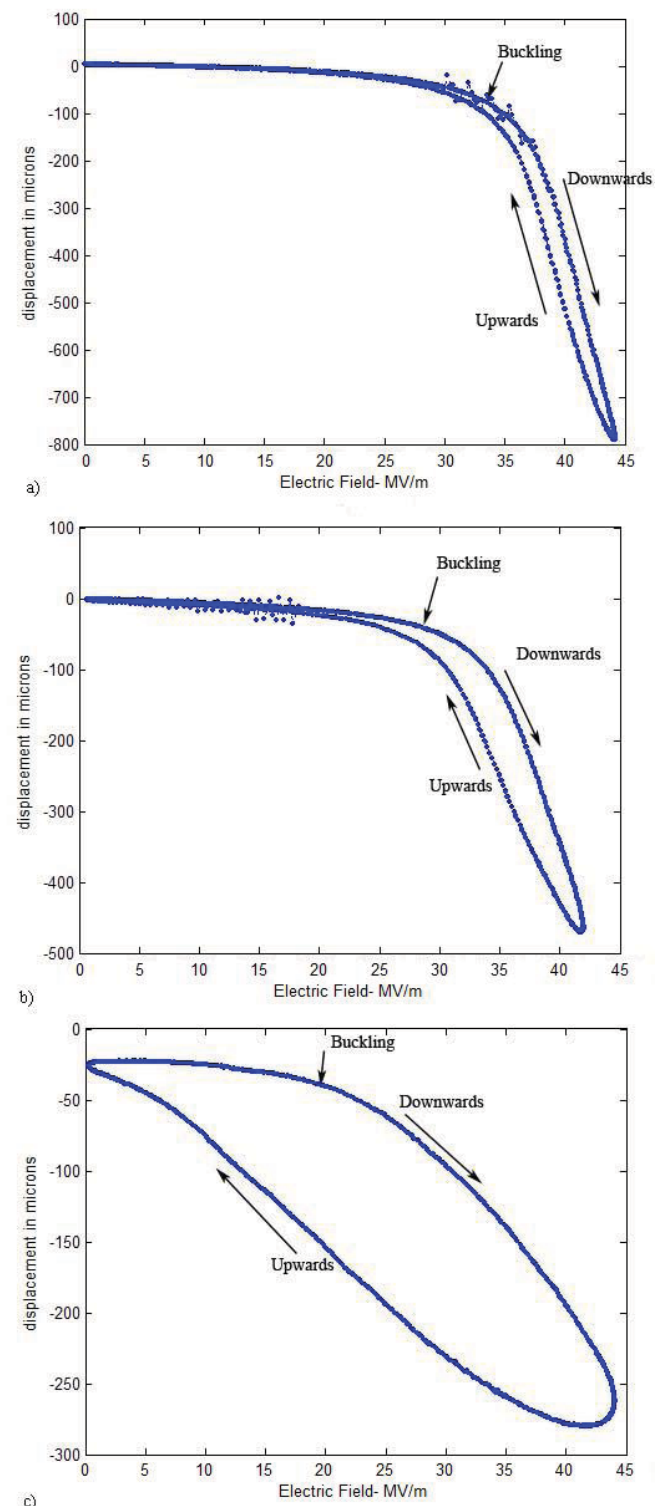

Figure 6: Out-of-plane deflection versus voltage for a) $15 \mathrm{~mm}, \mathrm{~b}) 10 \mathrm{~mm}$ and c) $5 \mathrm{~mm}$ radius dimple. 


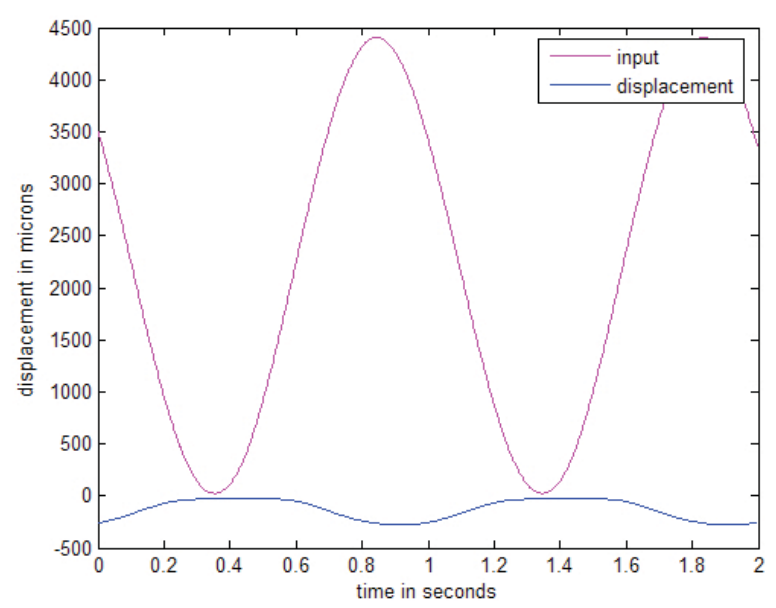

Figure 7: Out-of-plane behaviour for $5 \mathrm{~mm}$ dimple

\subsection{Frequency Response}

The dimple is designed to be flush with the surface when the driving voltage is zero and move into a depression when it is deployed. It is not desirable to excite dimples at, or beyond, resonance even though it is common to use resonance for improved efficiency. If the first resonant mode is excited, the dimple moves in both directions vertically. At higher harmonics, complex mode shapes are excited, as shown in Fig. 8.

The LDV results show three important features: (i) the resonant frequency decreases with increasing dimple size; (ii) the resonant frequency decreases with increasing voltage; and (iii) the feed line close to the electrode is not fully adhered and lifts during vibration which causes some asymmetry in the motion and degenerate modes. This can be seen for the last two vibrational modes, Fig. 8. Table 1 summarises the frequencies of the first four vibrational modes for all the dimple sizes.

Resonant frequency decreases with increasing voltage, as a result of the decrease in apparent stiffness of the film. The contribution from bending and stretching energies on the resonant frequency of the first mode vibration in an actuated circular PDMS actuator have been studied, [22]. It is found that by increasing the electrostatic stress there is a reduction in the stretching energy, resulting in an reduction in the resonant frequency. 

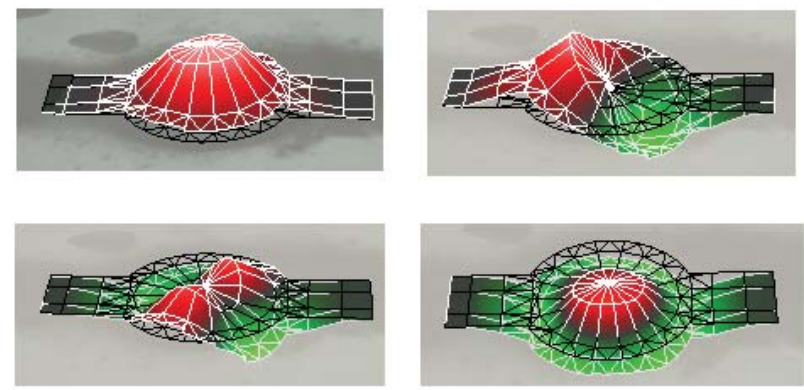

Figure 8: First four vibrational modes for a $5 \mathrm{~mm}$ dimple.

\begin{tabular}{|r|r|r|r|}
\hline $\begin{array}{r}\text { Loading } \\
\text { Vadius } \\
\hline\end{array}$ & $\begin{array}{l}\text { Dimple } \\
\text { Rm }\end{array}$ & \multicolumn{1}{l|}{$\begin{array}{l}\text { Vibrational } \\
\text { Modes }\end{array}$} \\
\hline 2000 & 5 & 01 & 405 \\
\hline & & 11 & 747 \\
\hline & & 21 & 1166 \\
\hline & & 02 & 1261 \\
\hline 3000 & 5 & 01 & 366.3 \\
\hline & & 11 & 677.5 \\
\hline & & 21 & 1091 \\
\hline & & 02 & 1179 \\
\hline 2000 & 10 & 01 & 201.2 \\
\hline & & 11 & 335.6 \\
\hline & & 21 & 504.6 \\
\hline & & 02 & 662.5 \\
\hline 3000 & 10 & 01 & 199.4 \\
\hline & & 11 & 329.4 \\
\hline & & 21 & 462.5 \\
\hline & & 02 & 503.8 \\
\hline 2000 & 15 & 01 & 131.9 \\
\hline & & 11 & 225 \\
\hline & & 21 & 298.8 \\
\hline & & 02 & 326.9 \\
\hline 3000 & 15 & 01 & 128 \\
\hline & & 11 & 290.6 \\
\hline & & 21 & 321.3 \\
\hline & & 02 & 492.4 \\
\hline
\end{tabular}

Table 1: Frequencies of the four lowest vibrational modes for the dimple sizes. 


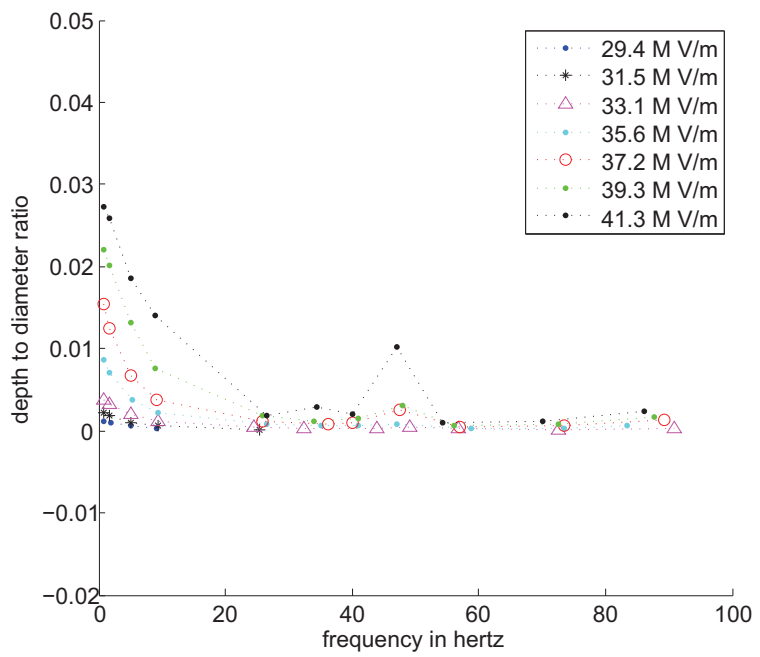

Figure 9: Depth-to-diameter ratio versus frequency for $15 \mathrm{~mm}$ radius dimple for different electric fields.

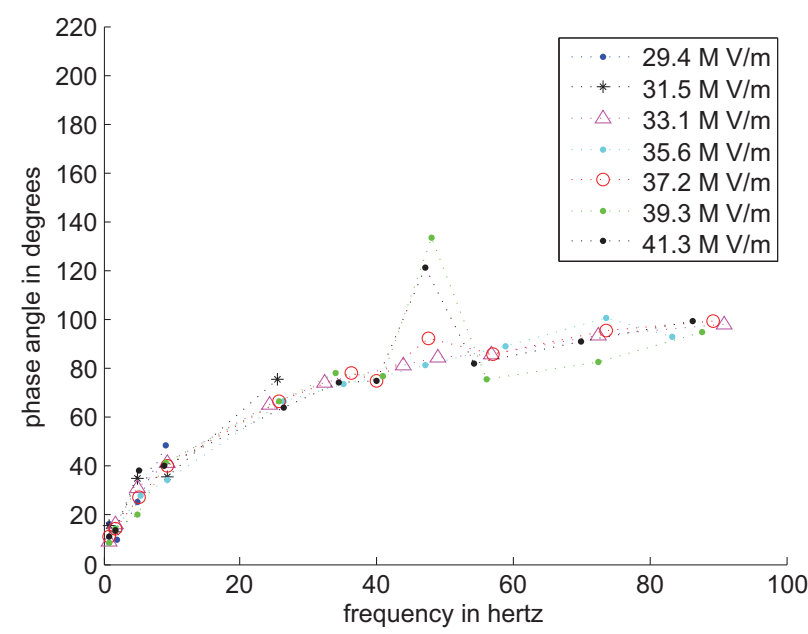

Figure 10: Phase angle versus frequency for $15 \mathrm{~mm}$ radius dimple for different electric fields. 


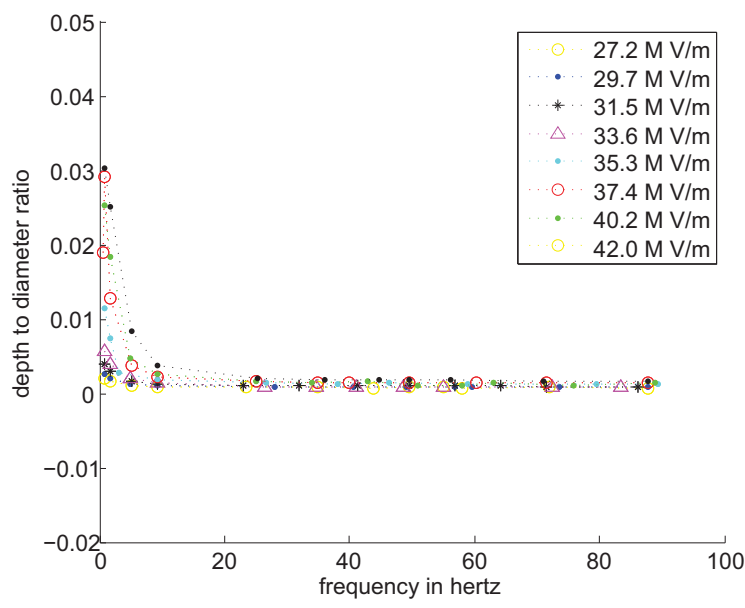

Figure 11: Depth-to-diameter ratio versus frequency for $10 \mathrm{~mm}$ radius dimple for different electric fields.

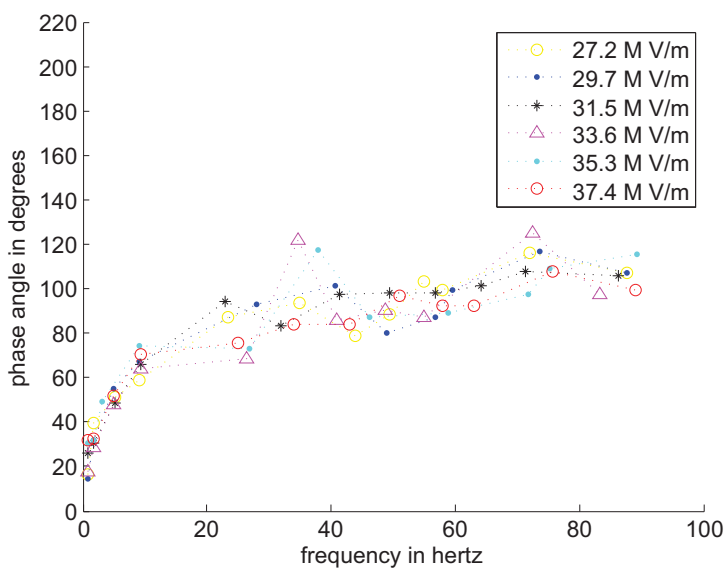

Figure 12: Phase angle versus frequency for $10 \mathrm{~mm}$ radius dimple for different electric fields. 


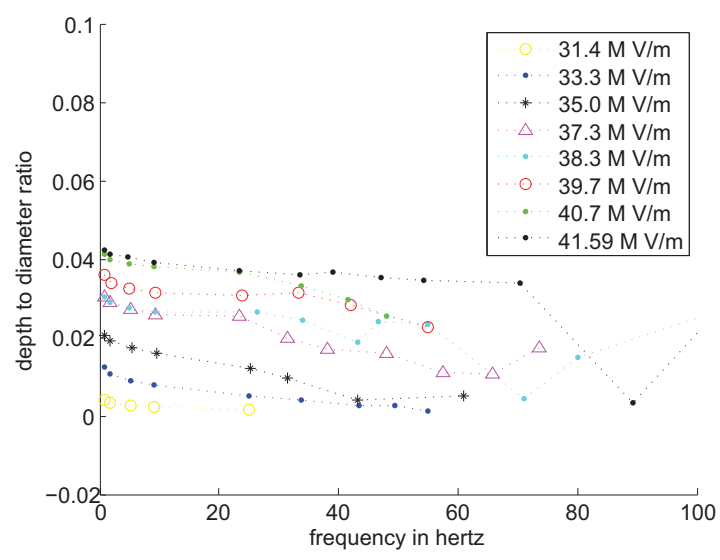

Figure 13: Depth-to-diameter ratio versus frequency for $5 \mathrm{~mm}$ radius dimple for different electric fields.

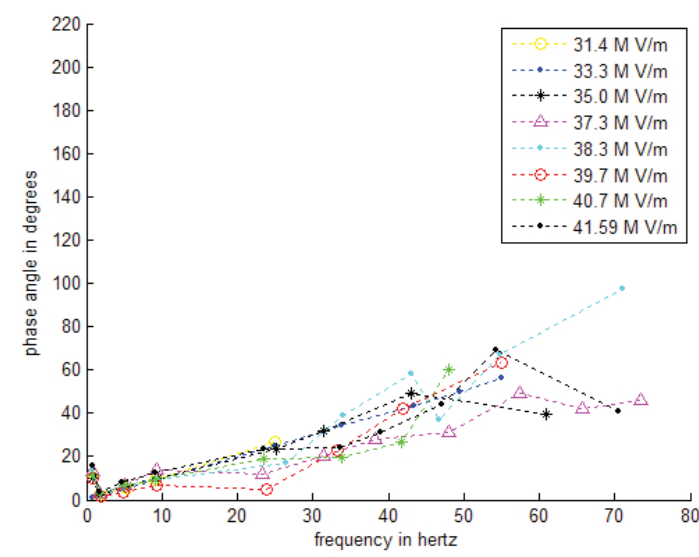

Figure 14: Phase angle versus frequency for $5 \mathrm{~mm}$ radius dimple for different electric fields. 


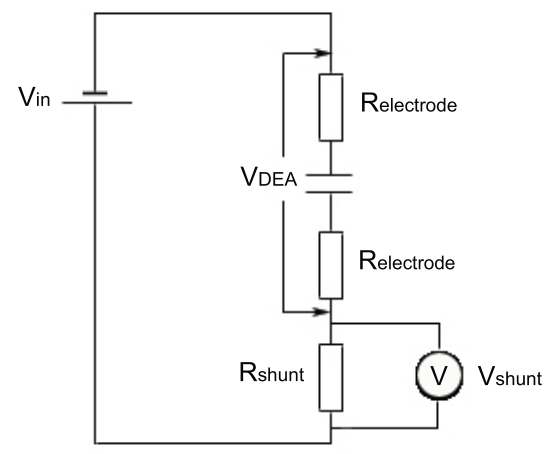

Figure 15: Circuit diagram for circuit measurements.

From Fig. 9, Fig. 11 and Fig. 13 it can be seen that, for larger dimples, deflections drop off rapidly as the frequency increases, whereas the performance of the 5-mm dimple is approximately constant. This means that it can be used at different frequencies at a constant amplitude (see $\S 5$ ). The amplitude of the 5 -mm dimple reduces at $60 \mathrm{~Hz}$ which is lower than the values of resonances reported by the LDV results.

The phase angles shown in Fig. 10, Fig. 12 and Fig. 14 describe the delay between the input signal and the dimple response. The depth-to-diameter ratio approaches zero for both the 15-mm and 10-mm dimples at approximately 20 Hz. At these frequencies, the phase angle approaches $90^{\circ}$ and increases with frequency. On the other hand, the phase angle of the 5-mm dimple does not increase above $90^{\circ}$ in the frequency range investigated and the performance of the dimple in this range is constant. Stiffness dominates the frequency response.

\subsection{Current and Power relationship}

In addition to size, amplitude and frequency response, the amount of energy consumed by the each sensor/actuator element needs to be considered. The total energy consumption by the entire control system has to be low enough to achieve net savings. Calculations by [9] put the power consumption of each sensor/actuator at $4,320 \mu \mathrm{W}$ in order to achieve savings of $60 \%$ of skin friction drag one metre from the leading edge. In order to work out the consumption for a dimple actuator it is necessary to calculate the current drawn. Current is measured via a shunt resistor, as shown in Fig. 15, the voltage across which is measured. For this purpose a voltage probe, LeCroy 
PPE66kV, with a bandwidth of $400 \mathrm{MHz}$ is selected and a shunt resistor of $22 \mathrm{kOhm}$. Using the shunt resistor, it is possible to calculate the total current across the circuit. Then using Kirchhoff and Ohm's law it is possible to calculate the current and power across the DEA alone. Thus the power consumed by a $5-\mathrm{mm}$ actuator in response to sinusoidal forcing at 1,10 , $100,1000 \mathrm{~Hz}$ is estimated as 450.0, 2,975, 25,010, 40,780 $\mu \mathrm{W} \pm 10 \mu \mathrm{W}(0.17$, 0.80, 6.67 and $10.9 \mu \mathrm{A}$ ) respectively. Larger dimples consume more power: for example the 15-mm dimple for forcing at 1, 10, 100, $1000 \mathrm{~Hz}$ consumes 160.0, 4,020, 56,000, 104,000 $\mu \mathrm{W} \pm 10 \mu \mathrm{W}$. These results are promising: devices would require miniaturisation in application, and then the capacitance and electrode area will decrease. Typically an actuator area would be one tenth of the present value.

\subsection{Actuator Failure}

Actuators will be liable to fail as a result of dielectric breakdown, whereby an electric field provides sufficient energy for free electrons to accelerate to high speeds, such that upon collision with other atoms, more electrons are knocked out, which in turn strikes other atoms. As this process continues, the number of free electrons moving through the material increases exponentially resulting in a short circuit and the burning of the dielectric membrane. This process is known as electron avalanche breakdown. Larger membranes are more prone to failure since they have a greater chance of material flaws and draw larger current. Defects acts as charge barriers accumulating charge such that the localised electric field exceeds the breakdown strength. The other mechanism for breakdown is caused by the presence of localised regions of negative charge, known as space charge, caused by Ohmic losses. Ohmic loss is the loss of electrical energy when a current flows through a resistance due to conversion of electrical energy to heat and is the cause of leakage current. In this case, the current that flows through the dielectric is small. Larger dimples draw larger current, §4.4, from the power supply, hence possess more leakage current across the dielectric, resultinh in excess free carriers, space charge, in the dielectric.

\section{Actuator Characteristics}

In the previous section, the characteristics of various dimple geometries have been evaluated. For the purpose of periodic forcing of a laminar boundary layer, the 5 -mm dimple is ideal, since it provides deformations that are 


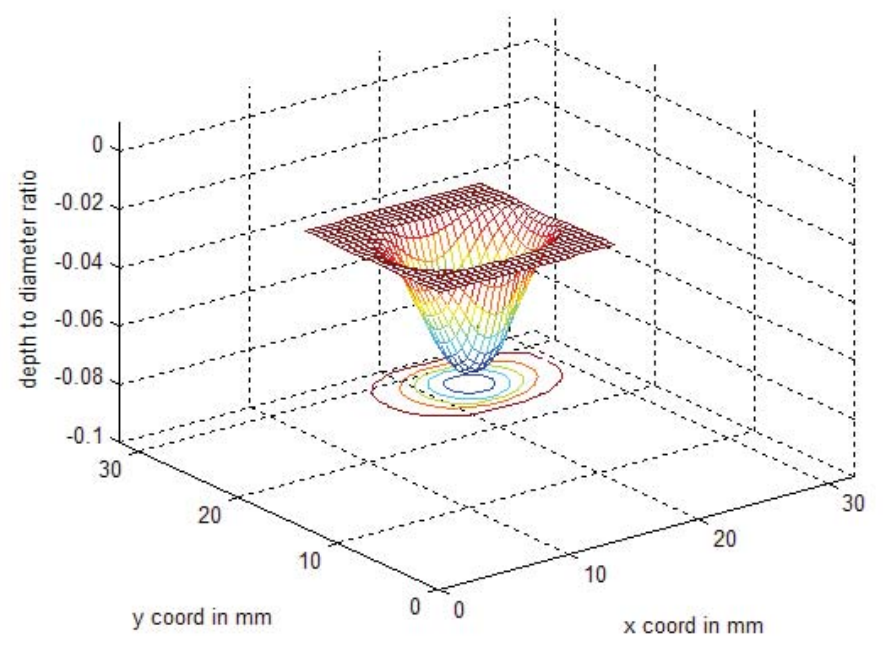

Figure 16: Dimple topology at maximum deflection for $5 \mathrm{~mm}$ dimple.

uniform and surfaces that are smooth, as shown from the topology of the dimple surface at maximum deflection oscillating at $1 \mathrm{~Hz}$, Fig. 16.

From the flow control perspective it is preferable to maintain a constant amplitude at all the frequencies under study so enabling a characterisation of the effects of surface acceleration, velocity and displacement separately. In Fig. 17 (b)- (d) displacement is measured using a laser meter and velocity and acceleration are calculated from numerical differentiation. It should be noted that the original displacement data are not noisy, the error being $\pm 1 \mu \mathrm{m}$. The temporal resolution of the displacement meter is $10 \mathrm{kHz}$. Calculating the propagation of error using the root sum of the squares, the error is low, less than $1 \%$ for both the velocity and acceleration calculations.

The traces shown in Fig. 17 (b)-(d) are described by the Strouhal number; for ease of comparison Table 2 shows the dimple frequency associated with the flow Strouhal numbers. The Strouhal number is a dimensionless frequency defined as: $S t=\frac{f \delta}{U_{\infty}}$, where $f$ is the frequency of actuation of the dimple, $U_{\infty}$ is the free-stream velocity of the boundary layer and $\delta$ is the boundary layer thickness.

Fig. 13 shows that 5-mm dimple has a fairly flat response up to $60 \mathrm{~Hz}$ and therefore a constant depth-to-diameter ratio at different frequencies can be maintained by marginally adjusting voltage. Fig. 17 (a) shows the displacement trace for one cycle at different frequencies: it can be seen that the 
peak displacement leads the driving voltage by approximately 1200. From this figure it can be seen that the displacement has been maintained at $400 \mu \mathrm{m}$ $\pm 50 \mu \mathrm{m}$.

\begin{tabular}{|l|l|}
\hline St & $\mathrm{f}(\mathrm{Hz})$ \\
\hline 0.006 & 5 \\
\hline 0.012 & 10 \\
\hline 0.025 & 21 \\
\hline 0.05 & 42 \\
\hline
\end{tabular}

Table 2: Table of conversions from Strouhal number to actuator frequency

An important feature for flow control purposes is the symmetry of the forcing. It is found that the upstroke and the downstroke of the dimple actuation show different behaviour. From Fig. 17 (a) it can be seen there is an asymmetry in dimple displacement: the dimple lies flush for a longer time in one cycle compared to the time it is buckled, even though the excitation signal is harmonic. This is a result of the actuator operating mechanism: the actuator spends more time flush to the surface than depressed as a result of the critical voltage required for the dimple to deflect. This asymmetry is reflected in velocity traces, which shows that the maximum and minimum velocities are different. The dimple velocity, Fig. 17 (c), shows an increase in magnitude with frequency. This asymmetry is accentuated in the acceleration trace, Fig. 17 (d), the peak acceleration occurs at maximum frequency. The asymmetry is pertinent to the way in which the boundary layer is forced.

\section{Conclusions}

Prototype dimple actuators have been designed, fabricated and tested in this study. These EAP devices use electrostatic which represents an alternative to current design which use external forces or mechanical asymmetries to control the direction of out-of-plane deflections. The performance of these devices is promising in terms of robustness, power consumption and out-of-plane deflections. This study has pinpointed various features of the actuator, pertinent to the overall objectives; specifically the presence of the higher modes of vibration and the asymmetrical forcing of the dimple on the fluid. To the authors' knowledge, such a complete characterisation of the diaphragm actuator has not been studied previously. While useful for flow control, these devices also provide a contribution to the actuator and sensor 

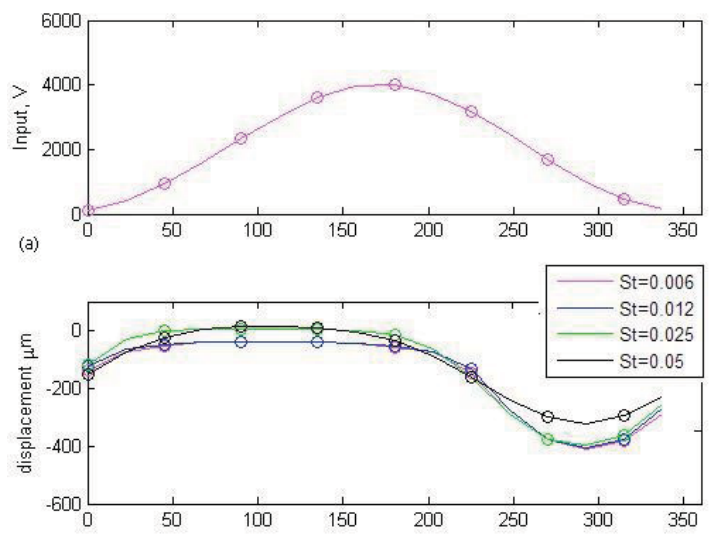

(b)

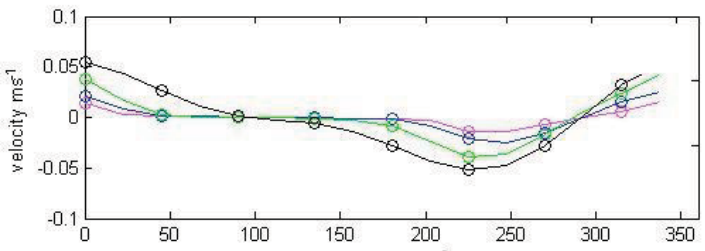

(c)

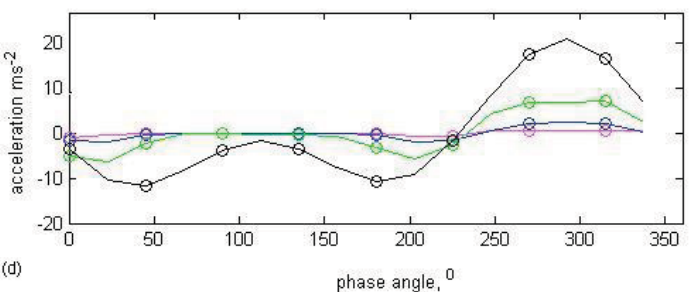

Figure 17: (a) Input Voltage, (b)Displacement, (c) Velocity and (d) Acceleration of a 5 $\mathrm{mm}$ radius dimple vibrating the various frequencies under study. 
community. At present, the prototype actuators do not have the size or frequency response required for turbulence control. However, the results of the evaluation suggest that robustness and frequency response will improve with miniaturisation, which is also noted in other studies $[21,32,14]$.

\section{Acknowledgments}

We are indebted to the Engineering and Physical Sciences Research Council for financial support (Grant GR/S20994/01). We are grateful to Graham Arthur of the Micro Nanotechnology Centre at the Rutherford Appleton (STFC) Laboratories for Figure 4.

\section{References}

[1] Morrison, J. F., Dearing S. S., Arthur, G. G., Cui Z., \& McKeon B. J., Fluid flow control using boundary layer control, International Patent Publication, (2006), WO 2006/040532 A1

[2] Dearing S. S., Lambert S. \& Morrison J. F., Flow control with active dimples, Aeronautical Journal, 3174 (2007), 705-714

[3] Arthur, G. G., Dearing S. S., McKeon, B., Cui, Z. \& Morrison J. F., Manufacture of Micro-sensors and Actuators for Flow Control, Microelectronic Engineering, 83 (2006),1205-1208

[13] Pelrine, R. E., Kornbluh, R. D. \& Joseph, J. P. Electrostriction of polymer dielectrics with compliant electrodes as a means of actuation, Sensors and Actuators A, 64, 1 (1998), 77-85

[5] Gad-el-Hak M., The Fluid Mechanics of Microdevices: The Freeman Scholar Lecture, J. Fluids Eng., 121(1997), 5-33

[6] McKeon, B. J., Lambert S., Sherwin, S. J. \& Morrison, J. F. , Active dimples for flow control, Proceedings of the 10th European Turbulence Conference, Trondheim, Advances in Turbulence X (2004), 581-584

[7] Bar-Cohen, Y., Electroactive polymers as artificial muscles reality, potential and challenges, SPIE Press (1998) 
[8] Bar-Cohen, Y., Shiuh Lih, S., \& Sheritt, S., Characterization of the electromechanical properties of EAP materials, Proceedings of SPIE: 8th Annual International Symposium on Smart Structures, Newport Beach, California, March 5-8, 2001, 4329(2001)

[9] Gad-el-Hak M., Innovative Control of Turbulent Flows, AIAA, 9332688 (1993)

[10] Sommer-Larsen, P., Hooker, J.C., Kofod, G., West, K., Benslimane, M. \& Gravesen, P. , Response of dielectric elastomer actuators Proceedings of SPIE: Smart Structures and Materials 2001: Electroactive Polymer Actuators and Devices, July 2001, 4329(2001), 157-163

[11] Pelrine, R., Kornbluh, R., Pei, Q. \& Joseph, J. , High-Speed Electrically Actuated Elastomers with Strain Greater Than 100\% Science,5454, 287(2000), 836-839

[12] Pelrine, R., Kornbluh, R., Joseph, J., Heydt, R., Pei, Q. \& Chiba, S., High-field deformation of elastomeric dielectrics for actuators, Materials Science and Engineering C, 2, 11 (2000), 89-100

[13] Pelrine, R., Kornbluh, R. \& Joseph, J., Electrostriction of polymer dielectrics with compliant electrodes as a means of actuation, Sensors and Actuators A, 64, 1 (1998), 77-85

[14] Dearing, S., Arthur, G., Morrison, J. \& Cui, Z., Electro-active polymers for flow control, II ECCOMAS Thematic Conference on smart structures and materials,, Lisbon, Portugal, July 18-21, 389(2005)

[15] Kofod, G., Dielectric elastomer actuators, Ph.D. thesis, The Technical University of Denmark (2001)

[16] Bao, X., Bar-Cohen, Y., Chang, Z. \& S. Sherrit, Numerical modeling of single-layer electroactive polymer mirrors for space applications, Proceedings of SPIE: Smart Structures and Materials 2003: Electroactive Polymer Actuators and Devices (EAPAD), 5051(2003), 381-388

[17] Goulbourne, N., Frecker, M. \& Mockensturm, E., Electro-elastic modeling of a dielectric elastomer diaphragm for a prosthetic blood pump Proceedings of SPIE: Smart Structures and Materials 2004: Electroactive Polymer Actuators and Devices (EAPAD), 5385(2004), 122-133 
[18] Tews, A., Pope, K. \& Snyder, A. , Pressure-volume characteristics of dielectric elastomer diaphragms Proceedings of SPIE: Smart Structures and Materials 2003: Electroactive Polymer Actuators and Devices (EAPAD), 5051(2003), 159-169

[19] Xu, Tian-Bing, Su, Ji \& Zhang, Qiming, Development, characterization, and theoretical evaluation of electroactive polymer-based micropump diaphragm, Sensors and Actuators A, 1, 121(2005), 267-274

[20] Lee, S. , Jung, K., Koo, J., Lee, S., Choi, H., Jeon, J., Nam, J. \& Choi, H., Braille display device using soft actuator Proceedings of SPIE: Smart Structures and Materials 2004: Electroactive Polymer Actuators and Devices (EAPAD), 5385(2004), 368-379

[21] Dubois, P., Rosset, S., Koster, S. , Stauffer, J., Mikhailov, S. , Dadras, M. , de Rooij, N. \& Shea, H., Microactuators based on ion implanted dielectric electroactive polymer (EAP) membranes Sensors and Actuators A, 130-131(2006), 147-154

[22] Dubois, P., Rosset, S., Niklaus, M. \& Shea, H., Voltage tuning of the resonance frequency of electroactive polymer membranes over a range of $75 \%$ Procedding of SPIE: Smart Sensors, Actuators, and MEMS IV, 7362, 73620F (2009).

[23] Löwe, C., Zhang, X., Kovacs, G., Wissler, M., Jähne, B., Dielectric elastomers in actuator technologies, Advanced Materials Engineering, $7(2005), 478$

[24] Wissler, M., Mazza E. \& Kovacs, G., Circular pre-strained dielectric elastomer actuator: modeling, simulation and experimental verification, Proceedings of SPIE: Smart Structures and Materials 2005: Electroactive Polymer Actuators and Devices (EAPAD), 5759(2005), 182193

[25] Wissler, M. \& Mazza E., Modeling of a pre-strained circular actuator made of dielectric elastomers Sensors and Actuators A, 1. 120(2005), 184-192

[26] Wissler, M. \& Mazza E., Modeling and simulation of dielectric elastomer actuators Smart Mater. Struct., 14(2005), 1396-1402 
[27] Heydt, R., Pelrine, R., R. Kornbluh \& V. Mason, Acoustical performance of an electrostrictive polymer film loudspeaker, Journal of Sound and Vibration, 2, 215(1998), 297-311.

[28] Zhang, X.Q., Wissler, M., Jaehne, B., Broennimann, R. \& Kovacs, G., Effects of Crosslinking, Prestrain and Dielectric Filler on the Electromechanical Response of a New Silicone and Comparison with Acrylic Elastomer, Smart Structures and Materials 2004: Electro-active Polymer Actuators and Devices (EAPAD), 5385(2004).

[29] Virlogeux, F., Bianchini, D., Delor-Jestin, F., Baba, M., \& Lacoste, J., Evaluation of cross-linking after accelerated photo-ageing of silicone rubber, Polymer International, 3(2)(2004), 163-168.

[30] Dearing, S., Electro-active Polymer(EAP), Dimple Actuators for Flow control, Ph.D. thesis, Imperial College(2007).

[31] Stein, M. \& Hedgepeth, J. M., Analysis of a partly wrinkled membrane, Tech. Rep. NASA, TND-813(1961).

[32] Pimpin,A., Suzuki, Y. \& Kasagi, N. Micro Electrostrictive Actuator With Metal Compliant Electrodes For Flow Control Applications, 17th IEEE Int. conf. MEMS 2004, Maastricht(2004), (2004), 478-484

[33] Boas,M. L. Mathematical methods in the physical sciences, Second Edition,John Wiley \& Sons, pp.209

[34] Young,W.,C., Roark's formulas for stress and strain, McGraw-Hill, New York, 6th ed. 1989 